\title{
Transverse Beam Spin Asymmetries in Forward-Angle Elastic Electron-Proton Scattering
}

D. S. Armstrong, ${ }^{1}$ J. Arvieux, ${ }^{2, *}$ R. Asaturyan, ${ }^{3, *}$ T. Averett, ${ }^{1}$ S. L. Bailey, ${ }^{1}$ G. Batigne, ${ }^{4}$ D. H. Beck, ${ }^{5}$ E. J. Beise, ${ }^{6}$ J. Benesch, ${ }^{7}$ L. Bimbot, ${ }^{2}$ J. Birchall, ${ }^{8}$ A. Biselli, ${ }^{9}$ P. Bosted, ${ }^{7}$ E. Boukobza, ${ }^{2,7}$ H. Breuer, ${ }^{6}$ R. Carlini, ${ }^{7}$ R. Carr, ${ }^{10}$ N. Chant,${ }^{6}$ Y.-C. Chao, ${ }^{7}$ S. Chattopadhyay, ${ }^{7}$ R. Clark, ${ }^{9}$ S. Covrig, ${ }^{10}$ A. Cowley, ${ }^{6}$ D. Dale, ${ }^{11}$ C. Davis, ${ }^{12}$ W. Falk, ${ }^{8}$ J. M. Finn, ${ }^{1}$ T. Forest, ${ }^{13}$ G. Franklin, ${ }^{9}$ C. Furget, ${ }^{4}$ D. Gaskell, ${ }^{7}$ J. Grames, ${ }^{7}$ K. A. Griffioen, ${ }^{1}$ K. Grimm, ${ }^{1,4}$ B. Guillon, ${ }^{4}$ H. Guler ${ }^{2}$ L. Hannelius, ${ }^{10}$ R. Hasty, ${ }^{5}$ A. Hawthorne Allen, ${ }^{14}$ T. Horn, ${ }^{6}$ K. Johnston, ${ }^{13}$ M. Jones, ${ }^{7}$ P. Kammel,${ }^{5}$ R. Kazimi, ${ }^{7}$ P. M. King, ${ }^{6,5}$ A. Kolarkar, ${ }^{11}$ E. Korkmaz,${ }^{15}$ W. Korsch, ${ }^{11}$ S. Kox,${ }^{4}$ J. Kuhn, ${ }^{9}$ J. Lachniet, ${ }^{9}$ L. Lee,,${ }^{8}$ J. Lenoble, ${ }^{2}$ E. Liatard, ${ }^{4}$ J. Liu, ${ }^{6}$ B. Loupias, ${ }^{2,7}$ A. Lung, ${ }^{7}$ D. Marchand, ${ }^{2}$ J. W. Martin, ${ }^{10},{ }^{16}$ K. W. McFarlane, ${ }^{17}$ D. W. McKee, ${ }^{18}$ R. D. McKeown, ${ }^{10}$ F. Merchez, ${ }^{4}$ H. Mkrtchyan, ${ }^{3}$ B. Moffit, ${ }^{1}$ M. Morlet, ${ }^{2}$ I. Nakagawa, ${ }^{11}$ K. Nakahara, ${ }^{5}$ R. Neveling, ${ }^{5}$ S. Ong, ${ }^{2}$ S. Page,${ }^{8}$ V. Papavassiliou, ${ }^{18}$ S. F. Pate, ${ }^{18}$ S. K. Phillips, ${ }^{1}$ M. L. Pitt, ${ }^{14}$ M. Poelker, ${ }^{7}$ T. A. Porcelli, ${ }^{15,8}$ G. Quéméner ${ }^{4}$ B. Quinn, ${ }^{9}$ W. D. Ramsay, ${ }^{8}$ A. W. Rauf, ${ }^{8}$ J.-S. Real, ${ }^{4}$ J. Roche,${ }^{7} 1$ P. Roos, ${ }^{6}$ G. A. Rutledge, ${ }^{8}$ J. Secrest, ${ }^{1}$ N. Simicevic, ${ }^{13}$ G. R. Smith, ${ }^{7}$

D. T. Spayde ${ }^{5,19}$ S. Stepanyan, ${ }^{3}$ M. Stutzman, ${ }^{7}$ V. Sulkosky, ${ }^{1}$ V. Tadevosyan, ${ }^{3}$ R. Tieulent, ${ }^{4}$ J. Van de Wiele, ${ }^{2}$ W. T. H. van Oers, ${ }^{8}$ E. Voutier, ${ }^{4}$ W. Vulcan, ${ }^{7}$ G. Warren, ${ }^{7}$ S. P. Wells, ${ }^{13}$ S. E. Williamson, ${ }^{5}$ S. A. Wood,${ }^{7}$ C. Yan, ${ }^{7}$ J. Yun, ${ }^{14}$ and V. Zeps ${ }^{11}$

\section{(G0 Collaboration)}

${ }^{1}$ Department of Physics, College of William and Mary, Williamsburg, Virginia 23187, USA

${ }^{2}$ Institut de Physique Nucléaire d'Orsay, CNRS/IN2P3, Université Paris Sud, Orsay, France

${ }^{3}$ Yerevan Physics Institute, Yerevan 375036 Armenia

${ }^{4} L P S C$, Université Joseph Fourier Grenoble 1, CNRS/IN2P3, INPG, Grenoble, France

${ }^{5}$ Loomis Laboratory of Physics, University of Illinois, Urbana, Illinois 61801, USA

${ }^{6}$ Physics Department, University of Maryland, College Park, Maryland 20472, USA

${ }^{7}$ Thomas Jefferson National Accelerator Facility, Newport News, Virginia 23606, USA

${ }^{8}$ Department of Physics, University of Manitoba, Winnipeg, MB R3T 2N2 Canada

${ }^{9}$ Department of Physics, Carnegie Mellon University, Pittsburgh, Pennsylvania 15213, USA

${ }^{10}$ Kellogg Radiation Laboratory, California Institute of Technology, Pasadena, California 91125, USA

${ }^{11}$ Department of Physics and Astronomy, University of Kentucky, Lexington, Kentucky 40506, USA

${ }^{12}$ TRIUMF, Vancouver, BC V6T 2A3 Canada

${ }^{13}$ Department of Physics, Louisiana Tech University, Ruston, Louisiana 71272, USA

${ }^{14}$ Department of Physics, Virginia Tech, Blacksburg, Virginia 24061, USA

${ }^{15}$ Department of Physics, University of Northern British Columbia, Prince George, BC V2N 4 Z9 Canada

${ }^{16}$ Department of Physics, University of Winnipeg, Winnipeg, MB R3B $2 E 9$ Canada

${ }^{17}$ Department of Physics, Hampton University, Hampton, Virginia 23668, USA

${ }^{18}$ Physics Department, New Mexico State University, Las Cruces, New Mexico 88003, USA

${ }^{19}$ Department of Physics, Grinnell College, Grinnell, Iowa 50112, USA

(Received 11 May 2007; published 29 August 2007)

We have measured the beam-normal single-spin asymmetry in elastic scattering of transversely polarized $3 \mathrm{GeV}$ electrons from unpolarized protons at $Q^{2}=0.15,0.25(\mathrm{GeV} / c)^{2}$. The results are inconsistent with calculations solely using the elastic nucleon intermediate state and generally agree with calculations with significant inelastic hadronic intermediate state contributions. $A_{n}$ provides a direct probe of the imaginary component of the $2 \gamma$ exchange amplitude, the complete description of which is important in the interpretation of data from precision electron-scattering experiments.

DOI: 10.1103/PhysRevLett.99.092301

PACS numbers: 25.30.Bf, 13.40.-f, 14.20.Dh, 24.70.+s

Elastic scattering of electrons from nucleons is usually treated in the single-photon exchange (Born) approximation. Higher order processes, such as two-photon exchange, are generally treated as small radiative corrections. However, interest in two-photon exchange was recently renewed when it was argued that contributions from the real part of this amplitude play a role in the discrepancy between the Rosenbluth separation and polar- ization transfer measurements of the ratio of the elastic form factors $G_{E}^{p} / G_{M}^{p}[1-3]$. In addition, although the twophoton exchange contribution is small, it is comparable to the parity-violating elastic electron-nucleon scattering asymmetry [4], and recent parity-violation measurements have had to consider possible systematic corrections due to this effect. A good understanding of two-photon exchange contributions can be extended to calculations of diagrams 
that appear in other processes, such as $\gamma Z$ and $W^{+} W^{-}$box diagrams, which are important corrections in precision electroweak experiments [5]. Thus, empirical verification of the theoretical framework for this effect is beneficial.

The two-photon exchange process involves the exchange of two virtual photons with an intermediate hadronic state that includes the ground state and all excited states, and it can produce a single-spin asymmetry in electron scattering [6]. The beam-normal single-spin asymmetry, or transverse asymmetry $A_{n}$, is sensitive to the imaginary part of the two-photon exchange amplitude in the elastic scattering of transversely polarized electrons from unpolarized nucleons, and it arises from the interference of the one-photon and two-photon exchange amplitudes [6]. Time-reversal invariance forces $A_{n}$ to vanish in the Born approximation, so it is of relative order $\alpha \approx \frac{1}{137}$. Furthermore, $A_{n}$ must vanish in the chiral limit and so it is suppressed by the ratio of the electron's rest mass to the beam energy, leading to an asymmetry on order of $10^{-5}-10^{-6}$ for $\simeq \mathrm{GeV}$ electrons. Hence, measurement of $A_{n}$ is challenging. For a beam polarized normal to the scattering plane, the transverse asymmetry is defined as $A_{n}=\frac{\sigma_{\dagger}-\sigma_{\downarrow}}{\sigma_{\uparrow}+\sigma_{\downarrow}}$, where $\sigma_{\uparrow}\left(\sigma_{\downarrow}\right)$ represents the cross section for the elastic scattering of electrons with spins parallel (antiparallel) to the normal polarization vector defined by $\hat{n} \equiv \frac{\left(\vec{k}_{e} \times \vec{k}^{\prime}\right)}{\left|\vec{k}_{e} \times \vec{k}^{\prime}\right|}$, where $\vec{k}_{e}$ and $\vec{k}^{\prime}$ are the three-momenta for the incident and scattered electron. The measured asymmetry $A_{\text {meas }}$ can be written as $A_{n} \vec{P}_{e} \cdot \hat{n}$. Because of the term $\vec{P}_{e} \cdot \hat{n}, A_{\text {meas }}$ is dependent on the azimuthal scattering angle $\phi$, which is manifested as a sinusoidal dependence in $A_{\text {meas }}$ versus $\phi$.

The transverse asymmetry due to two-photon exchange can be expressed using the formalism developed for the general amplitude for electron-nucleon elastic scattering [7]. This parametrization uses six complex functions, $\tilde{G}_{M}\left(\nu, Q^{2}\right), \tilde{G}_{E}\left(\nu, Q^{2}\right)$, and $\tilde{F}_{i}\left(\nu, Q^{2}\right), i=3, \ldots, 6$, dependent on $\nu$, the energy transfer to the proton, and $Q^{2}$, the four-momentum transfer. In the Born approximation, these functions reduce to the usual magnetic and electric form factors $G_{M}\left(Q^{2}\right), G_{E}\left(Q^{2}\right)$, and to $\tilde{F}_{i}=0$, so the $\tilde{F}_{i}$ and phases of $G_{M}, G_{E}$ must come from processes with the exchange of two or more photons. $A_{n}$ is proportional to the imaginary part of the combination of $\tilde{F}_{3}, \tilde{F}_{4}, \tilde{F}_{5}[8,9]$. Thus, $A_{n}$ is a function of $Q^{2}$ and the center-of-mass scattering angle $\theta_{\text {c.m. }}$, with the intermediate hadronic state information contained in the $\tilde{F}_{i}$.

There have been several calculations of the transverse asymmetry for the present kinematics [8-12], but the primary theoretical difficulty in calculations of the twophoton exchange amplitude is the large uncertainty in the contribution of the inelastic hadronic intermediate states. As the calculations require both the proton elastic form factors (elastic contribution) and the excitation amplitudes to other intermediate states, e.g., $\pi N$ (inelastic contribu- tion), experimental verification is important to test the framework of the calculations. However, at present experimental information on $A_{n}$ is scarce.

The first measurement of $A_{n}$ was performed by the SAMPLE Collaboration, at laboratory scattering angles of $130^{\circ} \leq \theta_{e} \leq 170^{\circ}$ at $Q^{2}=0.1(\mathrm{GeV} / c)^{2}$ with a $200 \mathrm{MeV}$ beam [13]. The PVA4 Collaboration recently reported measurements at somewhat higher beam energies at $30^{\circ} \leq \theta_{e} \leq 40^{\circ}$ and $Q^{2}=0.106,0.230(\mathrm{GeV} / c)^{2}$ [14]. The results from both indicate that models including only the nucleon elastic state are insufficient. Other preliminary data also suggest that the elastic contribution alone is insufficient [15].

The present measurement is at a higher beam energy $(3 \mathrm{GeV})$ and forward angle, where the $\pi N$ intermediate states are predicted to be a significant contribution to $A_{n}$ [8]. Furthermore, this beam energy falls in a transition range between models. At energies below the two-pion production threshold, the $\pi N$ intermediate state contribution can be calculated using pion electroproduction amplitudes based on experimental input. Above that limit, the $\pi N$ contribution is not well known, and there could be additional contributions to $A_{n}$ [8]. At very high energies and forward scattering angles (the diffractive limit), $A_{n}$ can be expressed simply in terms of the total photo-absorption cross section using the optical theorem $[10,16]$. For the present intermediate energy, corrections to the diffractive limit result have been calculated $[10,11]$.

We report a measurement of the beam-normal spin asymmetry $A_{n}$ from the elastic scattering of transversely polarized electrons from the proton at forward scattering angles using the apparatus for the G0 experiment [17] in Hall $\mathrm{C}$ at Jefferson Lab. The apparatus and the method of asymmetry extraction are described in Refs. [17-20]; only differences between the running conditions and analysis of the transverse asymmetry data and the parity-violation data are reported here.

A $40 \mu \mathrm{A}$ electron beam of energy $3.031 \mathrm{GeV}$ was generated with a strained GaAs polarized source [21]. The beam had a $32 \mathrm{~ns}$ pulse separation, to allow particle identification via time-of-flight (TOF). The beam helicity was held constant for $\frac{1}{30} \mathrm{~s}$ periods in sequences chosen pseudorandomly. Active feedback systems maintained helicity-correlated current and position changes below $\sim 0.3 \mathrm{ppm}$ and $10 \mathrm{~nm}$, respectively. A Wien filter precessed the spin of the longitudinally polarized beam electrons in the accelerator plane. The average longitudinal beam polarization was measured with a Møller polarimeter [22] for a range of spin precessions, and these data were fit to obtain the Wien setting for maximal transverse polarization in Hall $\mathrm{C}$ and to determine the magnitude of the polarization, $(74.3 \pm 1.3) \%$. The purely transverse nature of the beam was verified by a measurement of zero longitudinal polarization with the Møller polarimeter at the optimal Wien setting. 
The polarized electrons were scattered from a $20 \mathrm{~cm}$ long liquid hydrogen target [19]. Recoiling elastic protons were focused by a superconducting magnet onto eight arrays of segmented scintillation detectors arranged symmetrically in $\phi$ around the beam axis [18]. Each array consisted of 16 scintillator pairs used in coincidence to simultaneously measure the range of momentum transfers $0.12 \leq Q^{2} \leq 1.0(\mathrm{GeV} / c)^{2}$. The detectors were numbered in order of increasing values of $Q^{2}$. Detector events were sorted by TOF using custom time-encoding electronics [20].

Thirty hours of data were taken in the transverse beam configuration. The transverse asymmetry is not expected to vary rapidly with $Q^{2}$ in this kinematic region [12], so in order to improve statistical precision, the TOF spectra from the first 12 detectors in each array were summed into two spectra: $1-8$ and 9-12. These correspond to ranges of $0.12<Q^{2}<0.20$ and $0.20<Q^{2}<0.32(\mathrm{GeV} / c)^{2}$, with central values of $Q^{2}=0.15(\mathrm{GeV} / c)^{2}$ and $\theta_{\text {c.m. }}=20.2^{\circ}$, and $Q^{2}=0.25(\mathrm{GeV} / c)^{2}$ and $\theta_{\text {c.m. }}=25.9^{\circ}$, respectively. Data taken at higher $Q^{2}$ suffered from poor statistics and larger backgrounds, so results are not reported here.

The rates for the individual detectors were corrected for electronics deadtimes of $10 \%-15 \%$, giving an uncertainty of $0.05 \mathrm{ppm}$ in the asymmetries. False asymmetries due to residual helicity-correlated beam current, position, angle, and energy variations were calculated by linear regression to be $<0.12 \mathrm{ppm}$. No correction was applied, and a systematic uncertainty of $0.12 \mathrm{ppm}$ was adopted. A $\phi$-independent correction of, on average, $+2.29 \pm$ $0.59 \mathrm{ppm}$ was made to account for the large charge asymmetry $(\sim 570 \mathrm{ppm})$ of a small fraction $\left(\sim 10^{-3}\right)$ of the beam current with a 2 ns structure, "leakage beam" from beam intended for the other operating halls [18]. Uncertainties of $0.002 \mathrm{ppm}$ and $0.021 \mathrm{ppm}$ for $Q^{2}=$ $0.15,0.25(\mathrm{GeV} / c)^{2}$, respectively, arose due to the upper limit on the residual longitudinal polarization of the beam and the known parity-violating asymmetry [17]. No radiative corrections were applied [23].

Figure 1 shows a typical TOF spectrum; background extends on both sides of the elastic proton peak, consisting of quasielastic protons from the aluminum target windows and inelastic protons from both the aluminum and the hydrogen of the target. The measured asymmetry therefore consists of two components

$$
A_{\text {meas }}=f A_{\text {elas }}+(1-f) A_{\text {bkg }} \text {, }
$$

where $A_{\text {elas }}$ is the elastic asymmetry, $A_{\text {bkg }}$ is the background asymmetry contribution, and $f$ is the signal-tomeasured yield fraction. The TOF spectra were rebinned into several regions; fits over these TOF bin regions to both the yield and asymmetry in the region of the elastic peak were used in the background correction. The yield was modeled with a Gaussian elastic peak and a fourth-order polynomial background. The asymmetry was modeled us-

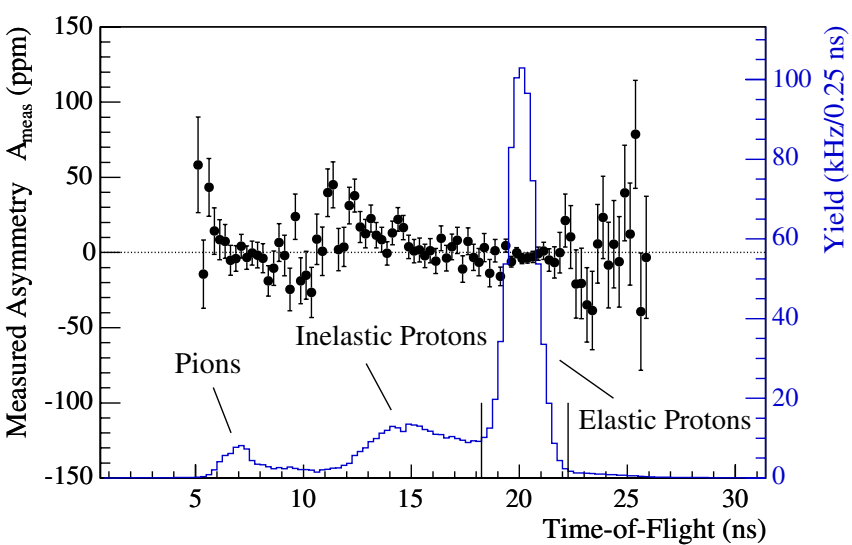

FIG. 1 (color online). Measured raw asymmetry, $A_{\text {meas }}$ (data points) and yield (histogram), as a function of the TOF for summed detectors $1-8$ in the array located at $\langle\phi\rangle=135^{\circ}$. Error bars are statistical.

ing a linear background and a constant for the elastic component. In a few cases, the asymmetry of the background was comparable in size to the measured asymmetry. As a check, the background asymmetries were also extracted using an alternate two-step fitting procedure in which the asymmetries from the background regions on either side of the elastic peak were used to interpolate the asymmetry from background processes beneath the elastic peak. This background asymmetry was then used to correct the elastic asymmetry, giving consistent results with the previous method. For a given azimuthal angle, the systematic uncertainty on the background correction was estimated from the dispersion of the extracted background asymmetries between the two methods.

The elastic transverse asymmetries, $A_{\perp}$ (the elastic asymmetry $A_{\text {elas }}$ corrected for all effects), for the eight detector arrays in each $Q^{2}$ bin are presented in Table I. The systematic errors are given in Table II; the error on the background correction clearly dominates and varies in $\phi$. A conservative model-dependent systematic error due to finite $Q^{2}$ bin size is indicated in Table II.

TABLE I. Elastic transverse asymmetries and uncertainties versus azimuthal scattering angle $\phi$. Uncertainties are statistical and individual systematic uncertainties, respectively (in Table II); global systematic uncertainties are not included.

\begin{tabular}{lrr}
\hline \hline & \multicolumn{2}{c}{ Elastic transverse asymmetries $A_{\perp}(\mathrm{ppm})$} \\
\hline$\phi$ & $Q^{2}=0.15(\mathrm{GeV} / c)^{2}$ & $Q^{2}=0.25(\mathrm{GeV} / c)^{2}$ \\
$0^{\circ}$ & $-0.38 \pm 1.94 \pm 0.62$ & $1.39 \pm 6.22 \pm 2.36$ \\
$45^{\circ}$ & $-1.15 \pm 1.84 \pm 0.49$ & $-1.09 \pm 3.35 \pm 1.09$ \\
$90^{\circ}$ & $-4.57 \pm 2.00 \pm 0.67$ & $-8.70 \pm 4.78 \pm 3.14$ \\
$135^{\circ}$ & $-4.39 \pm 2.04 \pm 0.76$ & $-1.67 \pm 3.18 \pm 0.87$ \\
$180^{\circ}$ & $-3.30 \pm 1.88 \pm 0.67$ & $-6.45 \pm 5.70 \pm 3.86$ \\
$225^{\circ}$ & $2.74 \pm 1.88 \pm 0.39$ & $5.68 \pm 3.33 \pm 0.83$ \\
$270^{\circ}$ & $2.25 \pm 2.00 \pm 0.71$ & $9.74 \pm 4.58 \pm 2.75$ \\
$315^{\circ}$ & $4.47 \pm 1.98 \pm 0.42$ & $1.13 \pm 3.06 \pm 0.81$ \\
\hline \hline
\end{tabular}


TABLE II. Systematic uncertainties in the asymmetries. The first three are point to point; the last four are global.

\begin{tabular}{lcc}
\hline \hline \multicolumn{1}{c}{ Source } & \multicolumn{2}{c}{ Uncertainty } \\
& $Q^{2}=0.15 \mathrm{GeV}^{2}$ & $Q^{2}=0.25 \mathrm{GeV}^{2}$ \\
\hline Dead time & $0.05 \mathrm{ppm}$ & $0.05 \mathrm{ppm}$ \\
False asymmetries & $0.12 \mathrm{ppm}$ & $0.12 \mathrm{ppm}$ \\
Background correction & $0.37-0.74 \mathrm{ppm}$ & $0.80-3.86 \mathrm{ppm}$ \\
Leakage beam & $0.55 \mathrm{ppm}$ & $0.63 \mathrm{ppm}$ \\
Beam polarization & $1.8 \%$ & $1.8 \%$ \\
Longitudinal polarization & $0.002 \mathrm{ppm}$ & $0.021 \mathrm{ppm}$ \\
Finite $Q^{2}$ binning & $0.03 \mathrm{ppm}$ & $0.03 \mathrm{ppm}$ \\
\hline \hline
\end{tabular}

The detector arrays are evenly spaced in azimuthal angle $\phi$ around the beam line, so the asymmetries should follow a sinusoidal dependence in $\phi, v i z$.

$$
A_{\perp}=\left|A_{n}\right| \sin \left(\phi+\phi_{0}\right)
$$

where the amplitude $\left|A_{n}\right|$ is the magnitude of the transverse asymmetry, and the phase $\phi_{0}$ depends on the direction of the transverse beam polarization. The electron polarization for the positive $(+)$ helicity is in the beam left direction. The azimuthal angle $\phi$ is defined to be $0^{\circ}$ on beam left and to increase clockwise as viewed along the direction of beam momentum. The data's sinusoidal dependence is displayed in Fig. 2, along with the best fit to Eq. (2). The

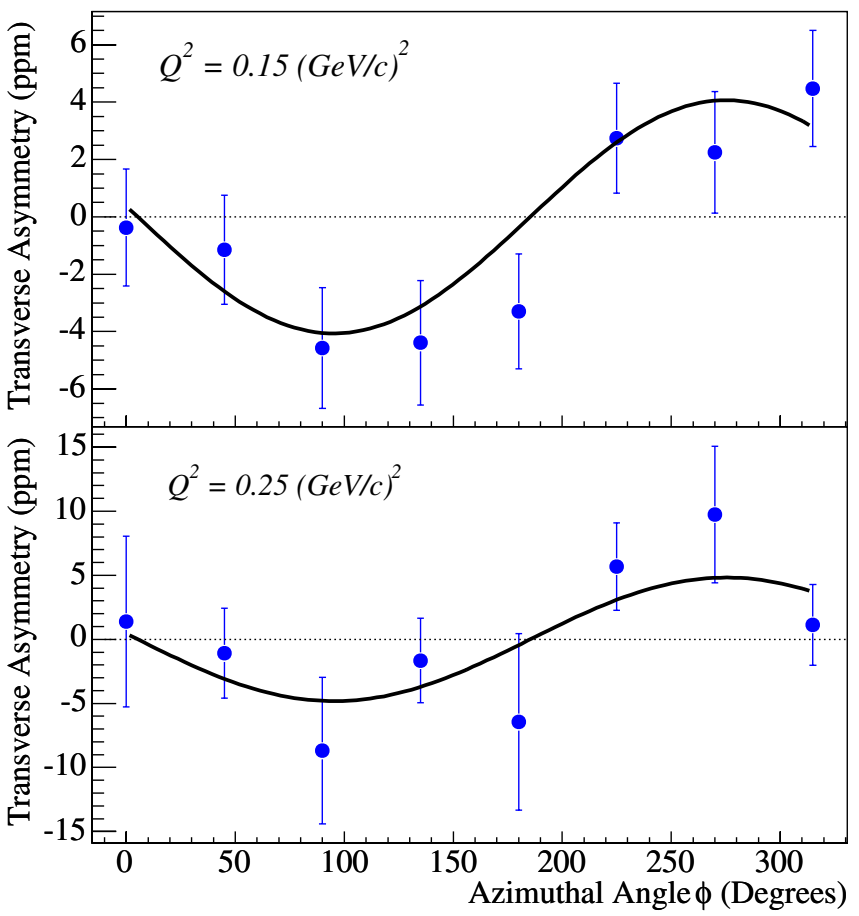

FIG. 2 (color online). Measured asymmetry as a function of the azimuthal scattering angle $\phi$ for $Q^{2}=0.15$ (upper plot) and $0.25(\mathrm{GeV} / c)^{2}$ (lower plot). The curves are the best fit to Eq. (2). Error bars are the statistical and individual systematic errors combined in quadrature. fits were constrained to $\phi_{0}$ as calculated from the electron spin precession in the $3 \mathrm{~T}$ solenoid used in the Møller polarimeter $\left(5.3^{\circ}\right)$ and do not allow a $\phi$-independent offset; however, relaxing these constraints has negligible impact on the extracted $A_{n}$. The small effect of finite bin size in $\phi$ was included.

Including all corrections, we obtain $A_{n}=-4.06 \pm$ $0.99_{\text {stat }} \pm 0.63_{\text {syst }} \mathrm{ppm}$ for $Q^{2}=0.15(\mathrm{GeV} / c)^{2}$ and $-4.82 \pm 1.87_{\text {stat }} \pm 0.98_{\text {syst }}$ ppm for $Q^{2}=0.25(\mathrm{GeV} / c)^{2}$ from the sinusoidal fits. Figure 3 compares these results to available calculations $[8,10,11]$ appropriate to these kinematics. The dash-double-dotted line [8] is a calculation of the two-photon exchange contribution solely from the nucleon intermediate state (elastic contribution); the dash-dotted line [8] represents the intermediate hadronic state for which the elastic contribution has been combined with inelastic contributions from excitation amplitudes to $\pi N$-intermediate states. The solid line [10] and the dashed line [11] represent calculations using the optical theorem and parametrizations for the measured total photoproduction cross sections on the proton. Clearly, the data show that the contribution of the inelastic hadronic intermediate states to the two-photon exchange amplitude is significant. This conclusion is consistent with those reported by SAMPLE [13] and PVA4 [14]; however, as the kinematics are different, the data points cannot be compared directly. The G0 experiment and other experiments [15] have recently obtained transverse beam spin asymmetry data at various angles on hydrogen, deuterium, and helium targets at additional $Q^{2}$ values, which will provide a further exploration of the imaginary part of the twophoton exchange amplitude.

The data reported here, along with other measurements, provide a valuable test of the theoretical framework of the two-photon contribution to the cross section through a comparison of the measured imaginary part of the twophoton exchange contribution to calculations of the real

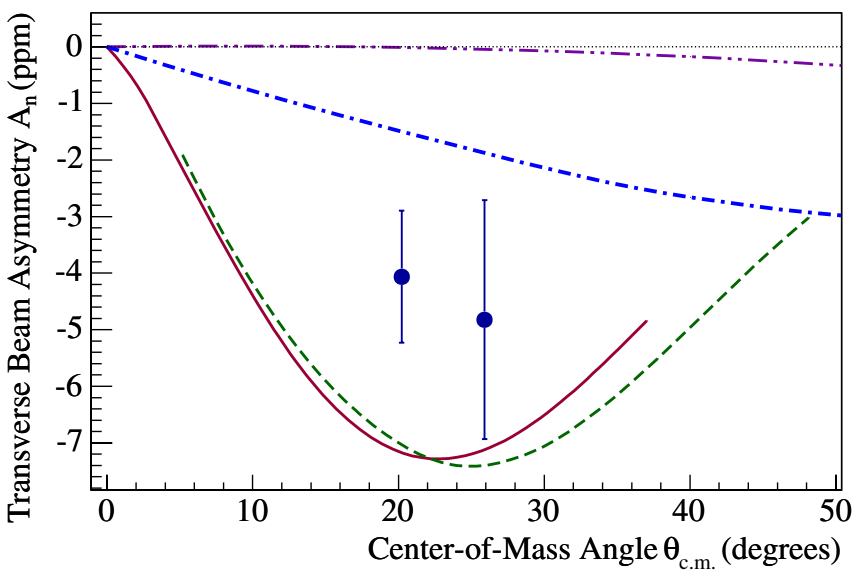

FIG. 3 (color online). Results for $A_{n}$ as a function of centerof-mass scattering angle, along with calculations from Refs. [8,10,11] (see text for explanation). 
part, as the two are related through dispersion relations [12]. These data underline the major role played by hadronic intermediate states in this process. Two-photon exchange and other box diagrams are important in the interpretation of high-precision parity-violating electronscattering experiments and in the radiative corrections for other lepton scattering experiments, making an understanding of these contributions important.

This work is supported in part by CNRS (France), DOE (USA), NSERC (Canada), and NSF (USA). We thank A. V. Afanasev, M. Gorchtein, B. Pasquini, and M. Vanderhaeghen for their calculations and useful discussions.

*Deceased.

[1] I. A. Qattan et al., Phys. Rev. Lett. 94, 142301 (2005).

[2] V. Punjabi et al., Phys. Rev. C 71, 055202 (2005); 71, 069902(E) (2005).

[3] P. A. M. Guichon and M. Vanderhaeghen, Phys. Rev. Lett. 91, 142303 (2003).

[4] A. V. Afanasev and C.E. Carlson, Phys. Rev. Lett. 94, 212301 (2005).

[5] W. J. Marciano and A. Sirlin, Phys. Rev. D 29, 75 (1984); 31, 213(E) (1985).

[6] A. De Rujula, J. M. Kaplan, and E. De Rafael, Nucl. Phys. B35, 365 (1971).

[7] M. L. Goldberger, Y. Nambu, and R. Oehme, Ann. Phys. (N.Y.) 2, 226 (1957).
[8] B. Pasquini and M. Vanderhaeghen, Phys. Rev. C 70, 045206 (2004).

[9] M. Gorchtein, P. A. M. Guichon, and M. Vanderhaeghen, Nucl. Phys. A741, 234 (2004).

[10] A. V. Afanasev and N. P. Merenkov, Phys. Lett. B 599, 48 (2004); arXiv:hep-ph/0407167v2.

[11] M. Gorchtein, Phys. Lett. B 644, 322 (2007).

[12] C.E. Carlson and M. Vanderhaeghen, arXiv:hep-ph/ 0701272.

[13] S. P. Wells et al. (SAMPLE Collaboration), Phys. Rev. C 63, 064001 (2001); E. J. Beise, M.L. Pitt, and D. T. Spayde, Prog. Part. Nucl. Phys. 54, 289 (2005).

[14] F. E. Maas et al. (A4 Collaboration), Phys. Rev. Lett. 94, 082001 (2005).

[15] L. Capozza, Eur. Phys. J. A 32, 497 (2007); L. Kaufman, Eur. Phys. J. A 32, 501 (2007).

[16] M. Gorshtein, Phys. Rev. C 73, 035213 (2006).

[17] D. S. Armstrong et al. (G0 Collaboration), Phys. Rev. Lett. 95, 092001 (2005).

[18] D.S. Armstrong et al. (G0 Collaboration) (to be published).

[19] S. D. Covrig et al., Nucl. Instrum. Methods Phys. Res., Sect. A 551, 218 (2005).

[20] D. Marchand et al., arXiv:nucl-ex/0703026 [Nucl. Instrum. Meth. Phys. Res. (to be published)].

[21] C. K. Sinclair et al., Phys. Rev. ST Accel. Beams 10, 023501 (2007).

[22] M. Hauger et al., Nucl. Instrum. Methods Phys. Res., Sect. A 462, 382 (2001).

[23] The correction is being investigated by A. V. Afanasev and N.P. Merenkov (private communication). 